\title{
Two-year follow-up of a randomized controlled trial of a clinical nurse specialist intervention, inpatient, and day patient team care in rheumatoid arthritis
}

\author{
Gerhardus J. Tijhuis MD \\ Department of Rheumatology, Leiden University Medical Center, Leiden, The Netherlands
}

Aeilko H. Zwinderman PhD

Department of Medical Statistics, Leiden University Medical Center, Leiden, The Netherlands

Johanna M.W. Hazes PhD

Department of Rheumatology, Leiden University Medical Center, Leiden, The Netherlands

Ferdinand C. Breedveld PhD

Department of Rheumatology, Leiden University Medical Center, Leiden, The Netherlands

and P.M. Theodora Vliet Vlieland PhD

Department of Rheumatology, Leiden University Medical Center, Leiden, The Netherlands

Submitted for publication 18 January 2002

Accepted for publication 7 October 2002

Correspondence:

Gerhardus Tijhuis,

Department of Rheumatology,

Leiden University Medical Center,

C4-R,

PO Box 9600,

2300 RC Leiden,

The Netherlands.

E-mail:g.j.tijbuis@lumc.nl

Present address:

Aeilko Zwinderman,

Department of Medical Statistics of the

Academic Medical Center of Amsterdam,

Amsterdam,

The Netherlands.

Present address:

Johanna Hazes,

Department of Rheumatology of the

University Hospital of Rotterdam,

Rotterdam,

The Netherlands.
TIJHUIS G.J., ZWINDERMAN A.H., HAZES J.M.W., BREEDVELD F.C. \& VLIET VLIELAND P.M.T. (2003) Journal of Advanced Nursing 41(1), 34-43

Two-year follow-up of a randomized controlled trial of a clinical nurse specialist intervention, inpatient, and day patient team care in rheumatoid arthritis

Aim. To compare the long-term effectiveness of care delivered by a clinical nurse specialist (CNS) with inpatient team care and day patient team care in patients with rheumatoid arthritis and increasing functional limitations.

Background. The role of CNSs in the management of patients with rheumatoid arthritis (RA) is evolving, and their effectiveness in comparison with care provided by a rheumatologist alone has been established. However, long-term controlled studies showing how the effectiveness of CNSs compares with that of other forms of co-ordinated care, such as multidisciplinary team care, are lacking.

Methods. Two hundred and ten patients rheumatoid arthritis patients were randomized to care delivered by a CNS in a rheumatology outpatient clinic (12 weeks), inpatient team care ( 2 weeks) and day patient team care ( 3 weeks). Clinical assessments recorded on study entry, weeks 12, 26, 52, 78 and 104 comprised the health assessment questionnaire (HAQ) and MacMaster Toronto Arthritis (MACTAR) patient preference interview as primary outcome measures. Grip strength, walk test, RAND-36, Rheumatoid Arthritis Quality of Life questionnaire and disease activity score (DAS) were applied as secondary outcome measures.

Results. No significant differences in medical treatment, use of services of other health professionals, introduction of adaptive equipment or number of hospitalizations were observed between the three treatment groups during 2 year follow-up, 
except that visits to nurse specialists were more frequent and home help was less frequent in the CNS group. A comparison of clinical outcomes among the three groups and a comparison between the nurse specialist and inpatient and day patient care groups together did not show any significant differences. Within all three groups functional status, quality of life and disease activity improved significantly $(P<0.05)$. In general, the results obtained after 12 weeks remained stable until 104 weeks after the start of the study.

Conclusion. Care provided by a CNS in an outpatient rheumatology clinic has a similar long-term clinical outcome to inpatient and day patient team care in patients with rheumatoid arthritis. A CNS intervention appears to be an effective innovation in the care for patients with rheumatoid arthritis.

Keywords: rheumatoid arthritis, chronic conditions, clinical nurse specialist, advanced nursing, nurse clinics, multidisciplinary team care, inpatient, day patient, randomized trial

\section{Introduction}

Rheumatoid arthritis (RA) is a chronic disease which may run a highly variable course, with periods of flares and remissions

\section{What is already known on this topic}

- The role of clinical nurse specialists in the management of patients with rheumatoid arthritis is evolving.

- The tasks of clinical nurse specialists may vary among institutions, ranging from substitution to addition of care provided by the rheumatologist.

- There has been a lack of long-term controlled studies which compare the effectiveness of clinical nurse specialists with the care provided by a multidisciplinary team, that being the treatment strategy that is considered to be optimal in complex chronic disease management.

\section{What this study adds}

- This randomized study showed that in patients with rheumatoid arthritis and functional deterioration over a period of two years, no sustained differences in clinical effectiveness were identified between care provided by a clinical nurse specialist in collaboration with a rheumatologist and that provided through inpatient or daypatient team care.

- The results of this study underscore that care provided by a clinical nurse specialist is a useful alternative to other available multidisciplinary management strategies for patients with rheumatoid arthritis in need of complex care. and steadily increasing damage to the joints. Despite new developments in the medical and surgical treatment, the disease has major physical, psychological, social and economic consequences in the majority of patients. Therefore, patients with RA often need long-term care from a wide variety of health care professionals and services. Research on health care utilization by patients with rheumatic diseases has revealed that they have a relatively high use of health care services in comparison with patients with other chronic conditions (Van den Bos 1995, Verbrugge \& Patrick 1995). In a recent Dutch study with 725 patients with RA (Jacobi et al. 2001) it was demonstrated that within the preceding 12 months $97 \%$ of the patients visited a rheumatologist, $42 \%$ a general practitioner, $23 \%$ an orthopaedic surgeon, $16 \%$ a rehabilitation specialist, $10 \%$ a plastic surgeon, $9 \%$ a neurologist, and $30 \%$ other specialists for RA purposes. Regarding allied health care, $40 \%$ of the patients made use of physiotherapy, $17 \%$ of occupational therapy, and $15 \%$ of chiropody. Furthermore, $18 \%$ had home help, and $4 \%$ nursing care at home. A social worker was visited by $10 \%$ of the patients, and $6 \%$ visited a psychologist or psychiatrist.

In case of progressive disability as a consequence of increasing disease activity, joint destruction, the occurrence of complications or comorbidity, complex interventions involving various health professionals at the same time are needed. Co-ordinated care by a team of health professionals in an inpatient or outpatient setting has long been considered an optimal form of health care in this situation (Davis et al. 2000). It is known from several randomized trials that inpatient multidisciplinary team care has a beneficial effect in patients with active RA in comparison with regular outpatient care, whereas the evidence for effectiveness of outpatient multidisciplinary team care in comparison with regular 
outpatient care is conflicting (Vliet Vlieland \& Hazes 1997). With respect to comparisons of different forms of team care programmes, Helewa et al. (1989) showed that inpatient team care appeared to be more effective than outpatient team care. Lambert et al. (1998) showed that the clinical outcome of team care provided in a day care setting proved to be equivalent to that of inpatient team care. Both studies included an economic analysis, showing that inpatient team care was more expensive than either outpatient or day patient team care.

Over recent years, limitations in the organization and availability of these services for large numbers of patients, and constraints on health care expenditures have created the need for new forms of complex health care. Over the last decade, clinical nurse specialists (CNSs) were introduced in many rheumatology practices, and they provide education, guidance and clinical care, and enhance and support care given by other health professionals as well (Bird et al. 1985).

Despite the wide adoption of CNSs in chronic disease management, evidence for their effectiveness is limited (Hobbs \& Murray 1999). Until 2001, only two studies with a controlled design had been conducted in the rheumatology setting (Hill et al. 1994, Hill 1997, Temmink et al. 2001). The content and organization of care provided by CNSs as described in these studies varied. In the study from the United Kingdom (UK) there was substitution of the rheumatologist's care by the rheumatology nurse specialist in a hospital setting (Hill et al. 1994, Hill 1997). In contrast, in the Dutch study the care provided by the CNS was given in addition to the usual care provided by the rheumatologist and other health professionals, under the joint responsibility of a hospital and home care organization (Temmink et al. 2001). In the study by Hill et al. it was shown that RA patients, reflecting a typical cross-section of the rheumatology outpatient population, who had had access to a CNS had significantly improved after 48 weeks with respect to pain, morning stiffness, psychological status, knowledge and satisfaction when compared with a group who received usual care provided by a consultant rheumatologist. In the Netherlands study, care provided by a CNS in addition to usual care provided by a rheumatologist did not influence patients' need for information, application of practical aids and adaptations, or functional capacity after 26 weeks in comparison with usual care provided by a rheumatologist. Neither of these studies included an economic analysis.

A direct comparison of the effectiveness of nurse specialist care with other forms of intensive, co-ordinated care in patients in need of complex care has only recently been carried out by our own group (Tijhuis et al. 2002). It was shown that care provided by a CNS was in the short term equally as effective as inpatient and day patient multidisciplinary team care However, the long-term outcome of CNS care remains to be established.

\section{The study}

\section{Aim}

The present follow-up study was undertaken to evaluate whether the similar improvement achieved by a short period of treatment by a CNS in comparison with inpatient team care and day patient team care could be maintained over a 2-year period.

\section{Methods}

Sample and ethical issues

Between December 1996 and January 1999 patients were recruited in the outpatient clinic of the Rheumatology Department of six hospitals. The inclusion criteria were RA as defined by the 1987 American Rheumatism Association criteria (Arnett et al. 1988) and increasing difficulty in performing activities of daily living over the last 6 weeks. Exclusion criteria were medical complications of RA requiring immediate hospitalization and inability to reach the hospital before 10 a.m. Random allocation was achieved by randomly assorted cards in sealed envelopes stratified by sex and centre. Ethical approval had been obtained for the study in all six participating hospitals. All patients gave written informed consent. To maintain allocation concealment, patients were asked not to inform blinded assessors about the type of care they received.

\section{Patient management protocols}

All patients randomized to care provided by a CNS were seen by a nurse specialist attached to the transmural nurse clinics of one of the six participating hospitals within 2 weeks after randomization. Transmural nurse clinics function under the joint responsibility of both the hospital and primary care organizations. The care provided by the CNSs was additional to the usual outpatient care provided by rheumatologists. The CNSs provided information about RA and prescribed, in consultation with the rheumatologist, joint splints, adaptive equipment and house adaptations if needed. If indicated, the patient could also be referred to other health professionals such as an occupational therapist, physical therapist or social worker. The time of termination of care by the CNS was left to their decision. The mean duration of care by the six CNSswas 12 weeks at the time the study was conducted and comprised on average three visits to the transmural nurse clinic. 
Multidisciplinary inpatient team care and day patient team care also started within 2 weeks after randomization and both were given at the rheumatology clinic of the Leiden University Medical Centre, a referral centre with inpatient and day care facilities. The multidisciplinary team in both the inpatient care and day care setting comprised nurses, a rheumatologist, an occupational therapist, a physical therapist and a social worker. Inpatients and day patients followed a prescribed treatment programme that was of equal intensity for both groups and tailored to individual needs. Treatment goals and modalities were discussed during weekly multidisciplinary team conferences. In addition, patients received written information about how to handle their disease and participated in a 1-hour educational session. Day care was given from 10 a.m. to 4 p.m., with a fixed period of $1 \frac{1}{2}$ hours of bed rest. Inpatient and day patient care both consisted of nine treatment days, given in a fixed period of 2 and 3 weeks, respectively.

Apart from the intervention period in the two team care groups, the decision to change or introduce disease-modifying drugs, optimize nonsteroidal anti-inflammatory drugs or administer intra-articular steroid injections was left to the rheumatologist at the outpatient clinic in all three study groups.

\section{Clinical assessments and outcome measures}

All assessments at study entry and at 12, 52, and 104 weeks of follow-up were carried out by two independent assessors who were blinded to the patient's treatment status. At baseline, a medical doctor (GJT) made an inventory of the medical history.

Clinical and socio-demographic characteristics recorded at baseline were age, disease duration, sex, rheumatoid factor, comorbidity (categorized as not present: Charlson index $=0$ and present: Charlson index of > 0) (Charlson et al. 1987), living status [living alone or living with other person(s)] and level of education (categorized as low: up to and including lower technical and vocational training; medium: up to and including secondary technical and vocational training; and high: up to and including higher technical and vocational training and university).

The independent assessors recorded the uptake of (para)medical services and introduction of practical aids and adaptations at weeks 26, 52, 78 and 104. Practical aids and adaptations were categorized as follows: home adaptations (heightened toilet or toilet seat, handles in the bathroom, shower chair, adjusted taps, adjusted windows and doors, alarm system, stair lift, adjusted kitchen, adjusted doorsteps, orthopaedic shoe(s) (adaptation), walking aids (stick, crutches, walking frame) and wheeled mobility aids [tipping chair (electric) wheelchair, scooter, adjustment to car]. Number of hospitalizations and use of home help and nursing care at home were recorded by means of a questionnaire filled in by the patient.

\section{Functional status}

Functional status, the primary outcome measure, was measured with the health assessment questionnaire (HAQ) (Siegert et al. 1984) and MacMaster Toronto Arthritis (MACTAR) patient preference interview (Tugwell et al. 1987). The HAQ contains 20 questions regarding eight domains of activities of daily living. The final score ranges from 0 (no disability) to 3 (severe disability). In the MACTAR, an interviewer assesses which activities are most impaired and most important (maximum 5) to the individual patient, and follows changes in these activities over time; it also includes questions on social and emotional functioning. Recently, the MACTAR has also been validated in Dutch patients (Verhoeven et al. 2000). Other (secondary) functional outcome measures included grip strength (mean grip strength of right and left hand) as measured by the Accoson ${ }^{\circledR}$ vigorimeter meter and walk test (time needed to walk a distance of 50 feet) (Grace et al. 1988).

\section{Quality of life}

Quality of life was measured with the RAND 36-item Health Survey 1.0 (RAND-36) (Hays et al. 1993) and Rheumatoid Arthritis Quality of Life (RAQoL) questionnaire (Jong de et al. 1997). The RAND-36 contains subscales for physical functioning, social functioning, role limitations (physical problem), role limitations (emotional problem), mental health, vitality, pain and general health perception. Each sub-scale generates a score from 0 to 100 , with higher score indicating better health. The RAND-36 may be converted to two summary scales: physical and mental component summary scales (Ware \& Sherbourne 1992). The RAND-36 includes the same items as the MOS-SF 36 and, although the scoring procedures are somewhat different, the effects of these on the scores are minimal (Hays et al. 1993). The RAQoL is a questionnaire consisting of 30 items with a yes/no (1/0) response format. It measures various areas of life, including moods and emotions, social life, hobbies, everyday tasks, personal and social relationships and physical contact. The overall score is the sum of the individual item scores, with a lower score indicating better quality of life. The RAND-36 and RAQoL have been both validated for use in the Netherlands (Zee van de \& Sanderman 1993, Jong de et al. 1997, Tijhuis et al. 2001). 
Table 1 Clinical and sociodemographic characteristics of 210 patients with rheumatoid arthritis and increasing difficulty in performing activities of daily living

\begin{tabular}{lccc}
\hline Characteristics & Nurse specialist $(n=71)$ & Inpatient $(n=71)$ & Day patient $(n=68)$ \\
\hline Age, years; median (range) & $54(24-85) *$ & $60(22-80)$ & $60(29-82)$ \\
Disease duration, years; median (range) & $2 \cdot 1(0-46)$ & $2 \cdot 1(0 \cdot 1-47)$ & $1 \cdot 4(0-35)$ \\
Number of patients (\%) & & & $54(79)$ \\
$\quad$ Women & $51(72)$ & $53(75)$ & $47(70)$ \\
With positive rheumatoid factor & $44(62)$ & $52(73)$ & $16(24)$ \\
With comorbidity & $23(32)$ & $26(37)$ & $15(22)$ \\
Living alone & $10(14)$ & $17(24)$ & $35(53)$ \\
With the following level of education & $33(46)$ & $44(62)$ & $24(35)$ \\
$\quad$ Low & $28(39)$ & $20(28)$ & $7(10)$ \\
$\quad$ Medium & $10(15)$ & $(12)$ & \\
High & &
\end{tabular}

*A significant difference between clinical nurse specialists' patients and day patients and inpatients (Mann-Whitney $U$-test, $P<0 \cdot 05$ ).

\section{Disease activity}

Disease activity was measured with the disease activity score (DAS) (Prevoo et al. 1995). This is a composite index of disease activity, including the number of tender joints, number of swollen joints, patient's global assessment of disease activity and erythrocyte sedimentation rate, according to the following formula: $0.555 \sqrt{ }$ (tender joints $)+0 \cdot 284 \sqrt{ }$ (swollen joints $)+0.70 \ln$ (erythrocyte sedimentation rate) +0.0142 (VAS patient's global assessment of disease activity).

\section{Data analysis}

To test clinical equivalence, the largest acceptable clinical difference in outcome between the groups was defined as $0 \cdot 22$ on the HAQ. A difference of $>0.22$ has been found to be clinically relevant (Redelmeier \& Lorig 1993). Based on a within-group standard deviation of 0.44 , a total sample size of 189 was required to detect this difference in the HAQ, between the unpaired groups, with a power of $80 \%$ at the $P<0.05$ level (Student's two tailed $t$-test). All analyses were based on intention to treat as initially assigned. All available data were used.

Measures with a Gaussian distribution are expressed as means and SD; otherwise, medians and ranges are presented. Patients' characteristics and use of medical and paramedical treatment during follow-up were compared using the Kruskal-Wallis and Mann-Whitney U-test or Pearson Chisquare test where appropriate. Within group differences in clinical outcome between baseline and follow-up scores, and between 12 and 52 weeks and 52 and 104 weeks were tested with the Wilcoxon signed rank matched-pairs test. To establish whether there were significant differences in clinical outcomes between CNS patients, inpatients and day patients, analysis of variance was performed on the change scores. In this analysis, adjustments for statistically significant differences at baseline regarding clinically relevant variables (sociodemographic and clinical variables and previous medical and paramedical treatment) were made by entering the baseline values of these variables as covariates into the analysis of covariance. The analysis was repeated with both types of team care (inpatient and day patient) taken together. Post hoc analysis was performed with Bonferroni correction for multiplicity.

\section{Results}

\section{Analysis of the sample}

Sixty of 270 patients who were screened for the study were not randomized for the following reasons: did not fit entry criteria (15), unwillingness to be randomized (10), private circumstances (21) and the expectation that day care would be too physically burdensome (14).

Thirty-one patients did not complete the study. They were equally distributed over the three groups. Seven of these 31 patients died. Other reasons for withdrawal were: severe comorbidity, deteriorating physical condition, unwillingness and removal. A comparison of baseline parameters between withdrawals and patients who completed 2-year follow-up showed that withdrawals were significantly older, had longer disease duration and more often had previous multidisciplinary treatment, and a higher percentage had a positive rheumatoid factor $(P<0.05)$.

During the initial treatment, one patient randomized to day care was hospitalized because travelling was considered physically too burdensome and one inpatient was hospitalized for 42 days instead of 9 days because of severity of RA activity. 
Table 2 Clinical outcome data at baseline [absolute values, means (SD)] and weeks 12, 52 and 104 [change scores from baseline, means (95\% confidence interval)] adjusted for age and differences at baseline

\begin{tabular}{|c|c|c|c|c|}
\hline & Baseline & Week 12 & Week 52 & Week 104 \\
\hline \multicolumn{5}{|l|}{ HAQ $(0-3)^{*}$} \\
\hline Nurse specialist patients & $1 \cdot 17(0 \cdot 65)$ & $0 \cdot 20(0 \cdot 07,0 \cdot 33)^{\mathrm{b}}$ & $0 \cdot 17(0 \cdot 03,0 \cdot 30)^{\mathrm{c}}$ & $0 \cdot 20(0 \cdot 06,0 \cdot 33)^{\mathrm{b}}$ \\
\hline Inpatients & $1.49(0.71)$ & $0 \cdot 15(0 \cdot 02,0 \cdot 27)^{\mathrm{b}}$ & $0 \cdot 19(0 \cdot 06,0 \cdot 32)^{\mathrm{b}}$ & $0 \cdot 13(-0 \cdot 03 \cdot 0 \cdot 26)^{\mathrm{c}}$ \\
\hline Day patients & $1.54(0.76)$ & $0.34(0.21,0.47)^{a}$ & $0.36(0.23,0.50)^{a}$ & $0 \cdot 35(0 \cdot 22,0 \cdot 48)^{\mathrm{a}}$ \\
\hline \multicolumn{5}{|l|}{ MACTAR weighted } \\
\hline Nurse specialist patients & $48 \cdot 4(3 \cdot 7)$ & $-2 \cdot 1(-4 \cdot 7,0 \cdot 5)$ & $-4 \cdot 3(-6 \cdot 8,-1 \cdot 8)^{\mathrm{b}, \mathbb{S}}$ & $-0 \cdot 6(-3 \cdot 3,2 \cdot 1)^{\curvearrowleft}$ \\
\hline Inpatients & $47 \cdot 2(3 \cdot 6)$ & $-0 \cdot 3(-2 \cdot 9,2 \cdot 4)$ & $0 \cdot 6(-2 \cdot 0,3 \cdot 1)$ & $0 \cdot 8(-2 \cdot 0,3 \cdot 6)$ \\
\hline Day patients & $47 \cdot 4(3 \cdot 7)$ & $-1 \cdot 5(-4 \cdot 2,1 \cdot 2)$ & $-5 \cdot 3(-7 \cdot 9,-2 \cdot 6)^{\dagger, \mathrm{a}, \mathbb{}}$ & $-0 \cdot 2(-3 \cdot 0,2 \cdot 5)^{\bullet}$ \\
\hline \multicolumn{5}{|c|}{ Grip strength* $(0-300, \mathrm{mmHg})$} \\
\hline Nurse specialist patients & $190(77)$ & $-17(-30,-5)^{\mathrm{c}}$ & $-23(-37,-9)^{\mathrm{b}}$ & $-18(-35,-2)$ \\
\hline Inpatients & $143(64)$ & $-23(-35,-11)^{\mathrm{b}}$ & $-31(-44,-17)^{\mathrm{a}}$ & $-32(-48,-15)^{\mathrm{a}}$ \\
\hline Day patients & $155(63$ & $-22(-34,-10)^{a}$ & $-34(-48,-21)^{\mathrm{a}}$ & $-21(-37,-4)^{c, \$}$ \\
\hline \multicolumn{5}{|l|}{ Walk test (seconds) } \\
\hline Nurse specialist patients & $12 \cdot 9(6)$ & $1 \cdot 5(0 \cdot 7,2 \cdot 3)^{\mathrm{b}}$ & $1 \cdot 7(0 \cdot 7,2 \cdot 7)^{\mathrm{a}}$ & $1 \cdot 1(0 \cdot 1,2 \cdot 1)$ \\
\hline Inpatients & $13 \cdot 5(6)$ & $1 \cdot 0(0 \cdot 2,1 \cdot 8)^{\mathrm{c}}$ & $1 \cdot 2(0 \cdot 2,2 \cdot 2)^{\mathrm{c}}$ & $0 \cdot 1(-0 \cdot 8,1 \cdot 1)^{\S}$ \\
\hline Day patients & $13 \cdot 7(7)$ & $1 \cdot 2(0 \cdot 4,2 \cdot 0)^{\mathrm{c}}$ & $1 \cdot 0(0 \cdot 0,2 \cdot 0)$ & $1 \cdot 2(0 \cdot 3,2 \cdot 2)^{\mathrm{c}}$ \\
\hline \multicolumn{5}{|c|}{ RAND Physical summary scale $(0-100) *$} \\
\hline Nurse specialist patients & $38 \cdot 0(21)$ & $-11 \cdot 4(-16 \cdot 9,-5 \cdot 9)^{\mathrm{a}}$ & $-15 \cdot 7(-21 \cdot 5,-9 \cdot 9)^{\mathrm{a}}$ & $-15 \cdot 1(-21 \cdot 0,-9 \cdot 2)^{\mathrm{a}}$ \\
\hline Inpatients & $29 \cdot 6(17)$ & $-7 \cdot 1(-12 \cdot 5,-1 \cdot 8)^{\mathrm{b}}$ & $-10 \cdot 4(-16 \cdot 0,-4 \cdot 8)^{a}$ & $-8 \cdot 5(-14 \cdot 3,-2 \cdot 8)^{\mathrm{b}}$ \\
\hline Day patients & $28 \cdot 2(20)$ & $-15 \cdot 6(-20 \cdot 9,-10 \cdot 2)^{\mathrm{a}}$ & $-15 \cdot 7(-21 \cdot 5,-9 \cdot 9)^{\mathrm{a}}$ & $-11 \cdot 2(-17 \cdot 0,-5 \cdot 4)^{a}$ \\
\hline \multicolumn{5}{|c|}{ RAND Mental summary scale $(0-100)^{*}$} \\
\hline Nurse specialist patients & $66 \cdot 3(24)$ & $-9 \cdot 3(-14 \cdot 7,-3 \cdot 9)$ & $-8 \cdot 6(-14 \cdot 5,-2 \cdot 7)$ & $-9 \cdot 9(-16 \cdot 1,-3 \cdot 5)$ \\
\hline Inpatients & $53.0(23)$ & $-4 \cdot 8(-10 \cdot 1,0 \cdot 5)$ & $-10 \cdot 6(-16 \cdot 2,-5 \cdot 0)^{\mathrm{a}}$ & $-6 \cdot 0(-12 \cdot 1,0 \cdot 1)^{\mathrm{b}}$ \\
\hline Day patients & $51 \cdot 3(26)$ & $-7 \cdot 1(-12 \cdot 4,-1 \cdot 8)^{\mathrm{b}}$ & $-9 \cdot 3(-15 \cdot 2,-3 \cdot 3)^{\mathrm{b}}$ & $-7 \cdot 3(-13 \cdot 4,-1 \cdot 2)^{\mathrm{b}}$ \\
\hline \multicolumn{5}{|l|}{ RAQoL $(0-30) *$} \\
\hline Nurse specialist patients & $13 \cdot 8(7)$ & $1 \cdot 0(-0 \cdot 4,2 \cdot 4)$ & $1 \cdot 7(0 \cdot 3,3 \cdot 1)^{\mathrm{c}}$ & $1 \cdot 9(0 \cdot 5,3 \cdot 3)$ \\
\hline Inpatients & $17 \cdot 0(6)$ & $1 \cdot 2(-0 \cdot 2,2 \cdot 6)$ & $1 \cdot 4(0 \cdot 1,2 \cdot 8)^{\mathrm{c}}$ & $0 \cdot 6(-0 \cdot 8,2 \cdot 0)$ \\
\hline Day patients & $18 \cdot 3(7)$ & $2 \cdot 3(0 \cdot 9,3 \cdot 7)^{\mathrm{a}}$ & $3 \cdot 1(1 \cdot 6,4 \cdot 5)^{\mathrm{a}}$ & $1.9(0 \cdot 5,3 \cdot 4)^{\mathrm{b}}$ \\
\hline \multicolumn{5}{|l|}{ Disease activity score $\$$} \\
\hline Nurse specialist patients & $5 \cdot 32(1 \cdot 24)$ & $0.7(0.4,0.9)^{\mathrm{a}}$ & $1.3(0 \cdot 9,1.6)^{\mathrm{a}, \boldsymbol{T}}$ & $1 \cdot 1(0 \cdot 8,1 \cdot 5)^{\mathrm{a}}$ \\
\hline Inpatients & $5 \cdot 72(1 \cdot 17)$ & $0.4(0.1,0 \cdot 7)^{\mathrm{b}}$ & $0.9(0 \cdot 6,1 \cdot 2)^{\mathrm{a}, \mathbb{S}}$ & $1 \cdot 0(0 \cdot 6,1 \cdot 3)^{\mathrm{a}}$ \\
\hline Day patients & $5 \cdot 85(1 \cdot 17)$ & $0.7(0.5,1 \cdot 0)^{\mathrm{a}}$ & $1.2(0.9,1.5)^{\mathrm{a}, \boldsymbol{\varpi}}$ & $0.9(0.6,1 \cdot 3)^{\mathrm{a}}$ \\
\hline
\end{tabular}

Significant improvement between admission and week 12, 52, and/or $104{ }^{\mathrm{a}} \mathrm{P}<0.001,{ }^{\mathrm{b}} \mathrm{P}<0 \cdot 01,{ }^{\mathrm{c}} \mathrm{P}<0.05$ (Wilcoxon signed rank matchedpairs test).

"Significant difference between clinical nurse specialists' patients versus day patients and nurse specialist patients versus inpatients at baseline (ANOVA, $P<0.01$ ).

${ }^{\dagger}$ Significant difference between day patients versus inpatients (Mann-Whitney $U$-test, $P<0 \cdot 01$ ).

'Significant difference between clinical nurse specialists' patients versus day patients at baseline (Mann-Whitney $U$-test, $P<0 \cdot 05$ ).

${ }^{\$}$ Significant difference(s) between weeks 12 and 52 and/or between weeks 52 and 104 (Wilcoxon signed rank matched-pairs test, $P<0 \cdot 05$ ).

"Significant difference(s) between weeks 12 and 52 and/or between weeks 52 and 104 (Wilcoxon signed rank matched-pairs test, $P<0 \cdot 01$ ).

\section{Clinical measures at baseline}

At baseline, no differences were found between CNS patients, inpatients and day patients with respect to clinical and socioeconomic characteristics, except that CNS patients were significantly younger than day patients and inpatients (Table 1).
At baseline, CNS patients had significantly better scores on the HAQ, RAND summary scales and RAQoL and higher grip strength than inpatients and day patients (Table 2). Furthermore, CNS ecialist patients had significantly lower disease activity than day patients. 


\begin{tabular}{|c|c|c|c|c|c|}
\hline & Weeks & Nurse patients & Inpatients & Day patients & $P$-value* \\
\hline \multirow[t]{2}{*}{ Starting with a new DMARD } & 6 & 12 & 22 & 18 & $0 \cdot 167$ \\
\hline & 12 & 5 & 5 & 1 & $0 \cdot 229$ \\
\hline \multirow[t]{2}{*}{ Started with oral prednisone } & 6 & 3 & 3 & 2 & $0 \cdot 886$ \\
\hline & 12 & 0 & 0 & 3 & $0 \cdot 051$ \\
\hline \multirow[t]{2}{*}{ Change of NSAID } & 6 & 12 & 15 & 14 & $0 \cdot 844$ \\
\hline & 12 & 12 & 9 & 8 & $0 \cdot 589$ \\
\hline \multicolumn{6}{|l|}{ Intra-articular steroid injections } \\
\hline \multirow[t]{2}{*}{ One or more in small joints } & 6 & 3 & 2 & 4 & $0 \cdot 684$ \\
\hline & 12 & 2 & 1 & 0 & $0 \cdot 363$ \\
\hline \multirow[t]{2}{*}{ One or more in large joints } & 6 & $6^{\dagger}$ & 16 & 17 & $0 \cdot 032$ \\
\hline & 12 & 11 & 9 & 6 & $0 \cdot 449$ \\
\hline
\end{tabular}

"Chi-squared test.

${ }^{\dagger}$ Significant difference between nurse patients versus inpatient and day patients (Mann-Whitney $U$-test).

\begin{tabular}{lrllll}
\hline \multirow{2}{*}{ Number of patients with one or more } & Weeks & $\begin{array}{l}\text { Nurse } \\
\text { patients }\end{array}$ & Inpatients & $\begin{array}{l}\text { Day } \\
\text { patients }\end{array}$ & $P$-value* \\
\hline Home adaptations & 52 & 29 & 20 & 22 & $0 \cdot 193$ \\
& 104 & 12 & 14 & 7 & $0 \cdot 269$ \\
Orthopaedic shoes/shoe adaptations & 52 & 27 & 22 & 27 & $0 \cdot 489$ \\
& 104 & 11 & 15 & 7 & $0 \cdot 203$ \\
Splint of wrist or knees & 52 & 29 & 34 & 31 & $0 \cdot 781$ \\
& 104 & 2 & 8 & 3 & $0 \cdot 090$ \\
Walking aids & 52 & 9 & 8 & 7 & $0 \cdot 864$ \\
Wheeled mobility aids & 104 & 4 & 3 & 7 & $0 \cdot 349$ \\
& 52 & 5 & 6 & 3 & $0 \cdot 609$ \\
& 104 & 3 & 3 & 3 & 0.999 \\
\hline
\end{tabular}

*Chi-squared test.

\section{Medical treatment and use of health services during the intervention period and 2-year follow-up}

With respect to medical treatment in the first 12 weeks of the study during which the interventions were completed in all three study groups, CNS patients received significantly fewer steroid injections in large joints than inpatients and day patients in the first 6 weeks $(P<0 \cdot 05)$ (Table 3). Over the first 12 weeks (Table 3), as well as during 104 weeks after the start of the study, no significant differences between CNS patients, inpatients and day patients were found with respect to the following treatment aspects: change of second line therapy, change of anti-inflammatory drug therapy, and oral use of prednisone (data not shown). Between weeks 12-52 and 52-104, no significant differences between CNS patients, inpatients and day patients were
Table 3 Medical treatment in clinical nurse specialists' patients, inpatients and day patients during first 12 weeks

Table 4 Introduction of practical aids and adaptations in clinical nurse specialist's (CNSs) patients, inpatients and day patients during 104 weeks follow-up, after the intervention found in the number of prescribed adaptive equipment and house adaptations (Table 4), number of patients hospitalized, and numbers who had one or more contacts with a physiotherapist, occupational therapist and/or social worker. In the period between 12 and 52 and 52-104 weeks, visits to a CNS were more frequent in the CNS group than in the inpatient and day patient groups $(P<0.05)$ (Table 5). Furthermore, in the period between 52 and 104 weeks more inpatients than CNS patients group received home help $(P<0.05)$ (Table 5).

\section{Clinical measures during 2-year follow-up}

During 2-year follow-up after the initial treatment all groups improved in functional status, quality of life and disease activity (Table 2). In general, no significant differences were 
Table 5 Numbers of patients with hospitalizations, receiving home help or using paramedical services in nurse patients, inpatients and day patients during 104 weeks follow-up, after the intervention

\begin{tabular}{lrrrcc}
\hline Number of patients & Weeks & Nurse patients & Inpatients & Day patients & $P$-value* \\
\hline Hospitalized & 52 & 5 & 10 & 9 & $0 \cdot 399$ \\
& 104 & 20 & 19 & 15 & $0 \cdot 644$ \\
Receiving home help & 52 & 9 & 21 & 17 & $0 \cdot 058$ \\
& 104 & $10^{\dagger}$ & 23 & 17 & $0 \cdot 029$ \\
Receiving nurse care at home & 52 & 3 & 3 & 4 & $0 \cdot 892$ \\
& 104 & 2 & 1 & 4 & $0 \cdot 317$ \\
Number of patients having one & & & & & \\
or more contacts with & & & & & \\
Physiotherapist & 52 & 41 & 46 & 40 & $0 \cdot 795$ \\
& 104 & 35 & 38 & 31 & $0 \cdot 681$ \\
Occupational therapist & 52 & 8 & 7 & 5 & $0 \cdot 699$ \\
& 104 & 2 & 4 & 2 & $0 \cdot 642$ \\
Social worker & 52 & 5 & 4 & 8 & $0 \cdot 382$ \\
& 104 & 3 & 3 & 5 & $0 \cdot 641$ \\
CNS & 52 & $22^{*}$ & 9 & 4 & $<0 \cdot 001$ \\
& 104 & 12 & 5 & 4 & $0 \cdot 045$ \\
\hline
\end{tabular}

*Chi-squared test.

${ }^{\dagger}$ Significant difference between nurse patients versus inpatients (chi-squared test, Mann-Whitney $U$-test).

${ }^{\ddagger}$ Significant difference between nurse patients versus inpatients and day patients (chi-squared test, Mann-Whitney $U$-test). observed between the results obtained between weeks $12-52$ and 52-104 in all three groups, except that between weeks 12 and 52 both CNS and day patients showed a further significant improvement in functional status as measured with the MACTAR, and in all three groups a significant improvement in disease activity was seen. Furthermore, between weeks 52 and 104 a significant deterioration was found on the MACTAR and grip strength scores in day patients, MACTAR scores in CNS patients, and walk test scores in inpatients.

With respect to differences between the three groups, the outcomes were very similar, except that day patients showed significantly greater improvement in functional status as measured with the MACTAR at week $52(P<0.01)$. A statistical comparison of change scores in the CNS group versus inpatient or day patient team care groups combined did not reveal any significant differences in outcome.

\section{Discussion}

This study shows that over a period of 2 years care provided by a CNS is equally as effective as inpatient and day patient multidisciplinary team care. In general, the improvement achieved by a short period of treatment remained stable over a period of approximately 104 weeks follow-up after the initial interventions.
The study had a randomized design and included two investigators who were blinded to patients' randomization status. The effectiveness was evaluated by use of several outcome measures commonly used in patients with RA, and patients were followed during a period of 2 years, which is much longer than in many other studies investigating these types of care (Hill et al. 1994, Hill 1997, Vliet Vlieland \& Hazes 1997, Temmink et al. 2001).

All patients in the study had RA and increasing difficulty in performing activities of daily living but were nevertheless able to be randomized between the three types of multidisciplinary care. Therefore, the results can be partly extrapolated to the general population of RA patients and deteriorating functional status, as for a number of these admission to hospital is inevitable.

In this randomized trial, care provided by a CNS was directly compared with multidisciplinary team care, which is generally considered to be an optimal strategy for patients with chronic diseases such as RA. The advantage of co-ordinated health care delivered by a team over nonteam care has been demonstrated in a systematic review on the role of patient care teams in chronic disease management (Wagner 2000). Despite these results, the present study shows that co-operation between a CNS and rheumatologist in combination with care provided by other health professionals results in an effective treatment regimen in which the clinical 
outcome does overall not differ from that of inpatient or day patient team care.

Differences in role of the CNS, study design, outcome measures and follow-up duration make comparison between two earlier studies (Hill et al. 1994, Hill 1997, Temmink et al. 2001) and the present study difficult. Patients who received care by a CNS also improved in the study by Hill et al. during a follow-up period of 48 and 104 weeks, respectively. These results contrasts with those of Temmink's study, in which no effect was observed. Possible explanations for differences in outcome may be the fact in Temmink's study not only RA patients were included, but more than $20 \%$ of the patients had other rheumatic diseases, and that patients were selected on the presence of 'problems regarding their disease', whereas in our study patients were included on the basis of having 'increasing functional limitations' during the last 6 weeks.

From several randomized trials it can be concluded that inpatient team care has a beneficial effect in patients with active RA in comparison with regular outpatient care (Lee et al. 1974, Vliet Vlieland et al. 1996). Regarding the comparison of inpatient team care with day patient team care, the results of the present study are in line with those of a previously published trial in which equivalent clinical outcomes were reported (Lambert et al. 1998). In contrast with that trial, the duration of the inpatient and day patient team care in the present study were exactly the same, confirming that staying overnight or for a weekend in hospital appears not to have an additional value in multidisciplinary team treatment. Furthermore, with regard to duration of benefit, our present findings are in accordance with the results of an earlier report by our group in which a positive effect of inpatient care continued during 2-year follow-up (Vliet Vlieland et al. 1997). This is in contrast with other studies (Helewa et al. 1989, Lambert et al. 1998) and may reflect differences in the intensity of regular outpatient management.

The present study showed a sustained effect for most outcome measures over the 2-year period after the start of the initial treatment, except for the MACTAR. Probably this questionnaire is less appropriate for measuring effectiveness over such a long period because with this tool activities that are most important to the individual patient at a certain time are evaluated, and these specific activities may not be considered that important or relevant after a longer period.

This study was performed with patients whose medical condition was such that it was acceptable for them to be randomized between all three types of care. Bearing this in mind, factors that may eventually play a role in the choice of treatment for patients with RA and functional limitations may be the presence of complications and comorbidity, availability of multidisciplinary facilities, patients' and doctors' preferences, and financial considerations.

Finally, this study demonstrates that in the management of patients with RA care provided by CNSs is a useful addition to other multidisciplinary approaches which have been available for a longer time, such as team care provided in an inpatient or day care setting. With respect to the process of innovation in complex disease management various chronic diseases are no longer being considered in isolation. It has been acknowledged that similar management strategies can be applied in different chronic conditions (Davis et al. 2000). Therefore, the results obtained with patients with RA may be relevant to therapeutic approaches to other chronic diseases, such as chronic obstructive pulmonary diseases, diabetes, coronary artery disease or neurological disorders. Management of these chronic diseases also consists of complex multidisciplinary treatment strategies which are nowadays more and more executed in outpatient and day care settings and in which CNSs are increasingly involved.

\section{Conclusion}

This study has demonstrated that in the management of patients with RA, care provided by the CNSs in transmural clinics is a useful addition to other multidisciplinary approaches, which have been available for a longer time, such as team care provided in an inpatient or day care setting.

\section{Acknowledgements}

We thank the following rheumatologists who participated in the study: J.M. Bok, Groene Hart Ziekenhuis, Gouda; Dr C. Mallée, Kennemer Gasthuis, Haarlem and J.Ph. Terwiel, Spaarne Ziekenhuis, Haarlem; Dr A.J. Peeters and Dr D. van Zeben, R. de Graaf Gasthuis, Delft; J.A.P.M. Ewals, Rode Kruis Ziekenhuis, Den Haag, G.A. Kok and Dr H.K. Ronday, Leyenburg Ziekenhuis, Den Haag, I. Speyer and Dr M.L. Westedt, Bronovo Ziekenhuis, Den Haag. In addition, we are indebted to all rheumatology nurse specialists from the participating hospitals. We wish to acknowledge I. Henkes and A.E.M. Jongma for doing the assessments and M. de Best-Bosz, H. Ravensbergen-van de Berg and A. van der Blom for secretarial assistance. This study was financially supported by Het Nationaal Reumafonds, The Dutch Arthritis Association, grant no. 931. 


\section{References}

Arnett F.C., Edworthy S.M., Bloch D.A., McShane D.J., Fries J.F., Cooper N.S. et al. (1988) The American Rheumatism Association 1987 revised criteria for the classification of rheumatoid arthritis. Arthritis and Rheumatism 31, 315-324.

Bird H.A., Le Gallez P. \& Hill J. (1985) Combined Care of the Rheumatic Patient. Springer-Verlag, Berlin.

Charlson M.E., Pompei P., Ales K.L. \& MacKenzie C.R. (1987) A new method of classifying prognostic comorbidity in longitudinal studies: development and validation. Journal of Chronic Disease 40, 373-383.

Davis R.M., Wagner E.G. \& Groves T. (2000) Advances in managing chronic disease. Research, performance measurement, and quality improvement are key. British Medical Journal 320, 525-526.

Grace E.M., Gerecz E.M., Kassam Y.B., Buchanan H.M., Buchanan W.W. \& Tugwell P.S. (1988) 50-foot walking time: a critical assessment of an outcome measure in clinical therapeutic trials of antirheumatic drugs. British Journal of Rheumatology 27, 372374.

Hays R.D., Sherbourne C.D. \& Mazel R.M. (1993) The RAND 36Item Health Survey 1.0 Health Economics 2, 217-227.

Helewa A., Bombardier C., Goldsmith C.H., Menchions B. \& Smythe H.A. (1989) Cost-effectiveness of inpatient and intensive outpatient treatment of rheumatoid arthritis. A randomized, controlled trial. Arthritis and Rheumatism 32, 1505-1514.

Hill J. (1997) Patient satisfaction in a nurse-led rheumatology clinic. Journal of Advanced Nursing 25, 347-354.

Hill J., Bird H.A., Harmer R., Wright V. \& Lawton C. (1994) An evaluation of the effectiveness, safety and acceptability of a nurse practitioner in a rheumatology outpatient clinic. British Journal of Rheumatology 33, 283-288.

Hobbs R. \& Murray E.T. (1999) Specialist liaison nurses. British Medical Journal 318, 683-684.

Jacobi C.E., Triemstra M., Rupp I., Dinant H.J. \& Van Den Bos G.A. (2001) Health care utilization among rheumatoid arthritis patients referred to a rheumatology center: unequal needs, unequal care? Arthritis and Rheumatism 45, 324-330.

Jong de Z., van de Heijde D.M.F.M., McKenna S.P. \& Whalley D. (1997) The reliability and construct validity of the RAQoL: a rheumatoid arthritis-specific quality of life instrument. British Journal of Rheumatology 36, 878-883.

Lambert C.M., Hurst N.P., Forbes J.F., Lochhead A., Macleod M. \& Nuki G. (1998) Is day care equivalent to inpatient care for active rheumatoid arthritis? Randomised controlled clinical and economic evaluation. British Medical Journal 316, 965-969.

Lee P., Kennedy A.C., Anderson J. \& Buchanan W.W. (1974) Benefits of hospitalization in rheumatoid arthritis. QJM 43, 205-214.

Prevoo M.L.L., Hof van 'T.M.A., Kuper H.H., Leeuwen van M.A., van de Putte L.B.A. \& Riel van P.L.C.M. (1995) Modified disease activity scores that include twenty-eight joint counts. Development and Validation. Arthritis and Rheumatism 38, 44-48.
Redelmeier D.A. \& and Lorig K. (1993) Assessing the clinical importance of symptomatic improvements. An illustration in rheumatology. Archives of Internal Medicine 153, 1337-1342.

Siegert C.E., Vleming L.J., Vandenbroucke J.P. \& Cats A. (1984) Measurement of disability in Dutch rheumatoid arthritis patients. Clinical Rheumatology 3, 305-309.

Temmink D., Hutten J.B.F., Francke A.L., Rasker J.J. \& Abu-Saad H.H. (2001) Rheumatology outpatient nurse clinics: a valuable addition? Arthritis Care Research 45, 280-286.

Tijhuis G.J., de Jong Z., Zwinderman A.H., Zuijderduin W.M., Jansen L.M., Hazes J.M. \& Vliet Vlieland T.P.M. (2001) The validity of the Rheumatoid Arthritis Quality of Life (RAQoL) questionnaire. Rheumatology 40, 1112-1119.

Tijhuis G.J., Zwinderman A.H., Hazes J.M.W., van den Hout W.B., Breedveld F.C. \& Vliet Vlieland T.P.M. (2002) A randomized comparison of care provided by a clinical nurse specialist, inpatient team care and day patient team care in rheumatoid arthritis. Arthritis Care and Research 47, 525-531.

Tugwell P., Bombardier C., Buchanan W.W., Goldsmith C.H., Grace E. \& Hanna B. (1987) The MACTAR Patient Preference Disability Questionnaire - an individualized functional priority approach for assessing improvement in physical disability in clinical trials in rheumatoid arthritis. Journal of Rheumatology 14, 446-451.

Van den Bos G.A.M. (1995) The burden of chronic diseases in terms of disability, use of health care and healthy life expectances. European Journal of Public Health 5, 29-34.

Verbrugge L.M. \& Patrick D.L. (1995) Seven chronic conditions: their impact on US adults' activity levels and the use of medical services. American Journal of Public Health 85, 173-182.

Verhoeven A.C., Boers M. \& van der Linden S. (2000) Validity of the MACTAR questionnaire as a functional index in a rheumatoid arthritis clinical trial. The McMaster Toronto Arthritis. Journal of Rheumatology 27, 2801-2809.

Vliet Vlieland T.P. \& Hazes J.M. (1997) Efficacy of multidisciplinary team care programs in rheumatoid arthritis. Semin Arthritis Rheum 27, 110-122.

Vliet Vlieland T.P., Zwinderman A.H., Vandenbroucke J.P., Breedveld F.C. \& Hazes J.M. (1996) A randomized clinical trial of in-patient multidisciplinary treatment versus routine out-patient care in active rheumatoid arthritis. British Journal of Rheumatology 35, 475-482.

Vliet Vlieland T.P., Breedveld F.C. \& Hazes J.M. (1997) The twoyear follow-up of a randomized comparison of in-patient multidisciplinary team care and routine out-patient care for active rheumatoid arthritis. British Journal of Rheumatology 36, 82-85.

Wagner E.H. (2000) The role of patient care teams in chronic disease management. British Medical Journal 320, 569-572.

Ware J.E. \& Sherbourne C.D. (1992) The RAND-36 Short-form Health Status Survey: 1. Conceptual frame work and item selection. Medical Care 30, 473-481.

Zee van de K.I. \& Sanderman R. (1993) Het meten van de algemene gezondheidstoestand met de RAND-36. (ISBN 90-72156-60-9). 